\title{
Accessibility Assessment of Prehospital Emergency Medical Services considering Supply-Demand Differences
}

\author{
Zhaoqing Shen, ${ }^{1}$ Ge Gao $\mathbb{D}^{1},{ }^{1}$ and Zhen Wang ${ }^{2}$ \\ ${ }^{1}$ College of Transportation, Shandong University of Science and Technology, Qingdao, China \\ ${ }^{2}$ Center for Big Data and Urban Space, Qingdao Urban Planning \& Design Research Institute, Qingdao, China \\ Correspondence should be addressed to Ge Gao; gaoge1@sdust.edu.cn
}

Received 2 July 2021; Accepted 27 September 2021; Published 21 October 2021

Academic Editor: Zhihong Yao

Copyright $(92021$ Zhaoqing Shen et al. This is an open access article distributed under the Creative Commons Attribution License, which permits unrestricted use, distribution, and reproduction in any medium, provided the original work is properly cited.

The reasonable accessibility assessment method is an important basis for the measurement of the level of prehospital emergency medical services. There is no general model for prehospital emergency care in traditional accessibility evaluation, and its supplydemand characteristics have also been ignored. Based on the three-step floating catchment area (3SFCA) model, the supplydemand three-step floating catchment area (SD3SFCA) model is proposed in this paper, which can express the difference between supply and demand of prehospital emergency medical services and accurately simulate unified dispatching of emergency centers. The unified dispatching behavior of emergency centers is simulated based on the potential service capacity of emergency stations with a supply-demand difference. The supply capacity of different emergency facilities is quantified from the perspective of infrastructure and technical quality. The needs of typical population densities are taken into account and adjusted by the weighting index. The validity of the model is verified, with the prehospital emergency medical service in the West Coast New District of Qingdao as an example. The results show that the model can effectively measure the accessibility level of prehospital emergency services and truly reflect the characteristics of supply and demand. Compared with previous models, the model has been significantly improved, which can provide an important reference for optimizing the allocation of prehospital emergency resources.

\section{Introduction}

Recent years have witnessed a surge in urban population density, the aging in urban areas, and backward urban management. These phenomena have given rise to a flurry of diseases and injuries such as various acute diseases, production accidents, traffic accidents, and natural disasters occurring more frequently. Therefore, how to rescue more efficiently in cases of injuries and diseases to minimize injuries has become a hot debated issue in the society. As a special medical service, the prehospital emergency medical service is designed for these acute critical diseases and sudden crises and its service directly determines whether patients can receive effective treatment at the first time when an injury occurs [1]. In China, the first aid center is endowed with the power to dispatch all the emergency resources in the region and it selects the most suitable emergency station to dispatch resources on receiving a call for help. Meanwhile, the first aid station receiving the dispatching instruction sends the first aid vehicle to the place where the injury occurs. The first aid center collects information on resource utilization and emergency events in the area, dispatching relevant resources with the purpose of global optimization. This omniscient perspective makes the resources-dispatched choice a completely rational behavior and proves to be a crucial step in the response process of emergency services. Presently, as the basis for optimizing the allocation of service resources, the accessibility evaluation of prehospital emergency medical services has increasingly become a research emphasis among scholars. However, most of the existing studies ignore the dispatch and selection process of emergency as well as the difference between supply and demand, which cannot objectively reflect the accessibility. Therefore, it is necessary to explore a more accurate and scientific 
evaluation method of urban prehospital emergency medical services in order to ensure that effective assistance is accessible to the injured.

The efficiency of prehospital emergency medical service is not only affected by distance but also closely related to the supply capacity and demand of facilities [2]. Due to the differences in population distribution and medical resource allocation, the method of evaluating prehospital emergency services based on the geographical location and coverage of emergency stations has been gradually eliminated and replaced by a comprehensive accessibility study. Accessibility study is used to analyze the spatial and temporal accessibility under a specific transportation system, turning out to be an important principle and tool to evaluate the availability of various public facilities and services. In addition, it can also be effectively used in the evaluation of prehospital emergency services [3]. With the help of the concept of accessibility, accurate evaluation and judgment of the prehospital emergency service can be achieved by establishing the relationship and restrictions among prehospital emergency medical services, injured patients, transportation systems, and spatial-temporal constraints.

However, existing studies on the accessibility of emergency medical services mainly focus on the influence degree and layout of the transportation network instead of providing sufficient quantitative research on the supply-demand difference of emergency services, which ignores the selection process of emergency centers with dispatching functions and cannot objectively reflect the working characteristics of prehospital emergency services. Therefore, 3SFCA is improved, and SD3SFCA is proposed in this paper, which can objectively quantify the difference between supply and demand of prehospital emergency medical services while considering the selection process of emergency centers with dispatching function, which is more in line with the characteristics of prehospital emergency services in real life. The supply-demand difference is reasonably quantified in the measurement of prehospital emergency medical service accessibility, and the selection probability is calculated to simulate the dispatching process of the emergency centers based on the potential service capacity of the emergency station. Through the accessibility analysis of prehospital emergency medical services in the West Coast New Area of Qingdao, the effectiveness and advancement of the improved method are verified, providing a universal fresh approach for measuring the accessibility of prehospital emergency medical services.

\section{Literature Review}

2.1. Accessibility. Accessibility has not yet formed a clear, unified definition. Scholars at home and abroad have given corresponding definitions and calculation methods for different research fields and objectives. Hansen has initially proposed the concept of accessibility, defined as the interaction opportunity of each node, reflecting the ease of approaching a certain point and measuring the accessibility of urban services [4]. The analysis of spatial accessibility is working as an effective method to evaluate the change in service capacity and spatial distribution fairness of public facilities. Many basic spatial accessibility measurement models have been proposed for different types of public services in the existing literature, and these models have different levels of data requirements and complexity based on different research objectives.

Classical spatial accessibility measurement models mainly include spatial barrier model, gravity model, opportunity model, and utility model. As the most intuitive model, the spatial barrier model is considered as the earliest model most widely used in the evaluation of accessibility of various public services. It is usually expressed by the travel time, distance, or cost between two nodes, which obtains facilitated calculation but lacks the expression of land use and other information [5]. In contrast, the gravity model reflects the attraction of the endpoint to the starting point through a certain degree of supply and demand [6]. Among the location-based accessibility measurement models, the opportunity model is most widely applied in planning practice, along with the measurement standards highlighting mainly the total opportunity available within a certain cost or the total cost required to obtain certain opportunities [7]. As the concept of accessibility is commonly used in many fields, some interdisciplinary methods have been proposed. For example, taking the discrete choice model as the theoretical basis, the utility model is recognized as a typical interdisciplinary model, which assumes that the individual chooses the travel destination according to utility. It is because of its interdisciplinary characteristics that the utility model still promises to be a model requiring tremendous research and further development [8].

With the development and integration of GIS, GPS, RS, and other related technologies, the application field of new methods innovated from the basic model is widely used in the service capacity evaluation of various public facilities [9]. These new models are used not only to consider travel characteristics and measure the convenience of residents to obtain public services in time and space [10] but also to pay attention to the constraints of nonspatial factors such as individual income and race on accessibility $[11,12]$. In terms of medical services, scholars have discussed accessibility assessment of medical facilities based on private cars, public transportation, and other modes of transportation combined with different models $[13,14]$. Among them, Tseng and $\mathrm{Wu}$ integrated socioeconomic status and spatial factors to access the accessibility of community-based care resources [15]. Wang and Gao focused on the spatial range of service accessibility under specific temporal and spatial constraints and calculated the accessibility by taking into account the differences in the time period, road segments, and direction of traffic conditions [16]. Significantly, in the relevant studies evaluating public transport-based accessibility to health facilities, Zhang et al. observed inaccurate evaluation results due to imbalanced spatial distribution of facilities and proposed a new approach considering the spatial heterogeneity [17]. However, unlike other public services, including general medical services, prehospital emergency medical services are for injured patients in critical emergency situations. This kind of service has higher requirements for response time and fairness, so the accessibility model in the 
emergency medical service pays more attention to the coverage within the golden first aid time and the satisfaction of first aid needs, rather than the discussion of spatial heterogeneity or access mode. Generally speaking, due to the complexity of the transportation network, different influencing factors, and spatial scales, as well as different characteristics of public service facilities, the evaluation methods of traffic accessibility also vary from each other. According to existing research, the subsequent improvement and deformation of the gravity-based 2SFCA is more appropriate for the assessment of prehospital emergency medical service levels owing to the consideration of the supply-demand relationship within the service threshold [18]. Therefore, this paper mainly adopts and improves the gravity-based search model to explore the accessibility evaluation model of the prehospital emergency medical service, which is more consistent with the characteristics of supply and demand difference.

2.2. Research Progress of the Gravity-Based Floating Catchment Model. On the basis of the gravity model, Luo and Wang first proposed a two-step floating catchment area (2SFCA) model, which overcomes the shortcomings of the traditional way by more accurately determining the effective catchment area of medical emergency service institutions [19]. When evaluating the accessibility of public services, the 2SFCA model with strong operability can take population distribution and resource allocation into consideration, widely used in the field of accessibility. However, in the model setting, the service facilities provide equal services within the service boundary, regardless of distance [20]. To remedy this defect, Luo and Qi have proposed the enhanced two-step floating catchment area (E2SFCA) model when studying the spatial accessibility of primary care physicians [21]. Compared with the basic method, the E2SFCA model divides the distance into three segments and assigns different distance weights to each segment. In order to make a more reasonable assumption of residents' demands for medical services, Wan and other scholars have proposed a three-step floating catchment area model (3SFCA model) [22] with a competitive scheme, adjusting the population demand by adding the selection weight of supply and demand points. Compared with previous methods, the 3SFCA model can effectively adjust the population demand and produce more reliable accessibility results for public service facilities.

With the continuous improvement of the floating catchment search model based on the gravity model, most research methods use various specified distance impedance coefficients to represent the impact of space-time distance on the effectiveness of public services. This coefficient determination method seems to be defective because different coefficient values will lead to the significant variations in the calculated value of spatial access index (SPAI). The spatial accessibility model based on gravity may result in apparent uncertainty in the analysis results; thus, some scholars believe that the spatial accessibility index calculated by various floating catchment models cannot well reflect the service level of public facilities. Wan et al. propose to replace SPAI with spatial access radio (SPAR) to present the calculation results of the E2SFCA model
[23]. Results show that although the SPAI of each community obtained by the E2SFCA model varies greatly with the change in distance impedance coefficient, the change in SPAR is relatively stable, which greatly reduces the uncertainty associated with the gravity-based spatial accessibility model. Having considered the supply and demand, Wan and others further propose the calculation method of adjusted spatial access (ASPA) and indicate that the corrected spatial access rate could quantitatively express the supply and demand ratio of public service facilities and play an important role in identifying the shortage areas of medical services [22]. From SPAI to SPAR, and then to ASPA, previous studies have planned to get a stable expression of accessibility with characteristics of supply and demand by complicated calculations and multiple comparisons. In contrast, based on the characteristics of first aid work, this study constructs an accessibility measurement model by using SPAI to evaluate the prehospital emergency medical service simply and intuitively.

Existing studies on the evaluation method of emergency medical services are inadequate, with more focus on the impact of road systems, lacking consideration for the expression of prehospital emergency dispatching choice behavior and the difference between supply and demand. In terms of supply capacity quantification, existing research methods mostly use a relatively large fixed value, or the number of hospital beds to represent the supply capacity of emergency stations, so as to obtain data easily. The fixed value cannot reflect the difference in rescue ability of emergency stations and lacks persuasion in quantitative supply, whereas, to a certain extent, the number of beds in a hospital can reflect the scale of the first aid station relying on the hospital. However, because the first aid vehicle is generally used as the carrier of prehospital first aid, the number of beds cannot fully demonstrate the supply capacity of the first aid station, and an overestimate of the supply capacity gap in the first aid station is more likely to appear. In the aspect of demand degree quantification, scholars mostly use the resident population data of each region to quantify the regional first aid demand and only a minority of studies touch on the demand difference under the influence of different age distributions in their application. This method can reflect the difference between supply and demand to a certain extent, but the consideration factor is single, showing no consideration for the potential demand of populationintensive points. Therefore, it is necessary to further analyze the dispatching choice behavior and service supply and demand of emergency centers in prehospital emergency medical services and establish the accessibility measurement method which can reasonably reflect the difference between supply and demand of prehospital emergency medical service.

\section{Construction of the SD3SFCA Model}

3.1. SD3SFCA Model. Fully taking into account the characteristics of emergency center's scheduling behavior and the supply-demand difference of prehospital emergency medical services, this study aims to construct the supplydemand three-step floating catchment area (SD3SFCA) 
model, a measurement model of prehospital emergency medical service accessibility considering the difference between supply and demand by improving the floating catchment area model on the basis of supply and demand and the spatial relationship between them. The improved model is implemented in three steps.

Step 1. Calculating the dispatching selection probability of emergency centers. According to the widely promoted concept of a 15-minute first aid circle, the maximum catchment area of the demand point $i$ is determined within the 15-minute driving time of ambulances taken as the effective threshold. Eight minutes is internationally recognized as the golden time for first aid, which is the optimal rescue time range for a variety of acute emergencies. Therefore, the maximum catchment area is divided into two subareas with an interval of 8 minutes. The first catchment subarea is within driving time of $0-8$ minutes from the demand point $i$, and the second one is within $8-15$ minutes. Meanwhile, the area beyond the driving time of 15 minutes is regarded as the invalid emergency area where the emergency station is not selected. Equation (1) is used to calculate the probability of the emergency center $\operatorname{Pro}_{i j}$ to select and dispatch the emergency station $j$ within the catchment area $i$ when $i$ generates the emergency demand.

$$
\operatorname{Pro}_{i j}=\frac{\left(S_{j} / P_{j}\right) \cdot f\left(d_{i j}\right)}{\sum_{k \in\left(d_{i k} \leq d_{0}\right)}\left(S_{k} / P_{k}\right) \cdot f\left(d_{i j}\right)},
$$

where $S_{j}$ and $P_{j}$ represent the supply capacity of the emergency station $j$ and the potential demand within its service scope, respectively. $S_{k}$ and $P_{k}$ represent the supply capacity of the emergency station within the catchment area $k$ and the potential demand within its service scope, respectively. The ratio of supply capacity to potential demand of each emergency station represents its potential service capacity, which is an important measure of emergency center selection and dispatching and whose calculation method will be discussed later. $d_{0}$ is the threshold travel time within a 15 -minute interval, $d_{i j}$ is the temporal distance from the demand point $i$ to the supply point $j$ in the catchment area, and $f\left(d_{i j}\right)$ is the time distance decay function determined by the time distance $d_{i j}$ from the demand point $i$ to the emergency station $j$ according to the distance decay function, representing the influence of time distance on the potential service ability of the emergency site. The first catchment subarea within 8 -minutes is regarded as the initial impedance without decay, and the Gaussian function curve descends slowly near the origin, which is more consistent with the influence of distance decay in the process of emergency medical response. Thus, the Gaussian function is modified to represent the influence of distance decay.

$$
f\left(d_{i j}\right)= \begin{cases}1 & d_{i j} \leq 8 \\ e^{-\left(d_{i j}-8\right)^{2} / \beta} & 8<d_{i j} \leq 15 \\ 0 & d_{i j}>15\end{cases}
$$

where $\beta$ is the impedance coefficient, whose value directly affects the distance decay of prehospital emergency medical services, so we should pay more attention to its value setting. The next section will discuss the determination of its value setting.

Step 2. Calculating the supply-demand ratio $R_{j}$ of the emergency station $j$. The catchment area division of the emergency station $j$ is similar to the first step, which starts from point $j$ to search the demand point $l$ within 15 minutes. The demand degree of all demand points in the catchment area is given by the corresponding distance decay $f\left(d_{j l}\right)$ according to the time distance $d_{j l}$ from $j$ to $l$. The calculation method of supply-demand ratio $R_{j}$ of the emergency station $j$ is shown as follows:

$$
R_{j}=\frac{S_{j}}{\sum_{l \in\left(d_{j l} \leq d_{0}\right)} \operatorname{Pro}_{l j} P_{l} f\left(d_{j l}\right)},
$$

where $\operatorname{Pro}_{i j}$ is the selection probability of demand point $l$ and emergency station $j$ and $P_{l}$ is the demand degree of demand point $l$. The method for determining $f\left(d_{j l}\right)$ is similar to the first step, which is obtained by the modified time distance decay function. The calculation method of supply and demand in the first and second steps will be discussed later in this section.

Step 3. Calculating SPAI $A_{i}$ of each demand point $i$. SPAI $A_{i}$ can be computed through the method of starting from demand point $i$. We search all the first aid stations that can provide first aid services within 15 minutes and sum up their supply and demand.

$$
A_{i}=\sum_{j \in\left(d_{i j} \leq d_{0}\right)} \operatorname{Pro}_{i j} R_{j} f\left(d_{i j}\right),
$$

where $A_{i}$ is SPAI calculated by the model, which directly reflects the accessibility of prehospital emergency medical services. The higher the value is, the greater the level of prehospital emergency medical services is.

3.2. Selection of the Impedance Coefficient. Figure 1 shows the change in distance decay function $f\left(d_{i j}\right)$ under different coefficient values $\beta$. The first search subarea is designed in the range of 0 to 8 minutes where the prehospital emergency medical service is almost not affected by distance decay, so the function value is determined as 1 . When $d_{i j}$ is involved in the range of 8 to 15 minutes, it can be seen from observing the change trend of each function image that no matter what the coefficient value is, the distance decay function decreases sharply with the increase in time distance, which represents the characteristic that the accessibility of prehospital emergency medical services decreases with the increase in time distance. It can be seen from the numerical difference of the distance decay function of different coefficient values $\beta$ at the same time distance that the value $\beta$ has an obvious influence on the distance decay function. With the increase in the distance, the value of the distance decay function gradually increases and approaches the value 1; i.e., the 


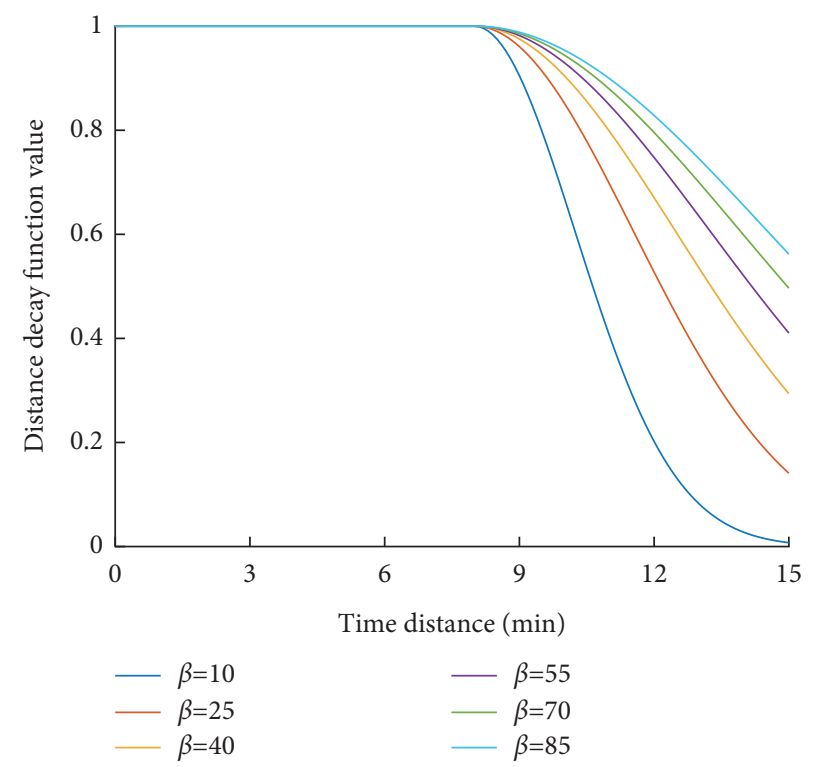

FIgURE 1: The distance decay function with different impedance coefficients.

decrease in the impedance coefficients represents a relatively strong impedance. Therefore, it can be concluded that the more obvious the value $\beta$ is, the stronger the influence of time distance on accessibility is. Considering emergency medical service as a special medical service which is extremely sensitive to time, it is not appropriate to set it too large when determining the value. As is discussed by Kwan [24], when the Gaussian function value is close to 0 and 0.01 , it is a critical value. For the 15-minute emergency, $\beta$ equals 10 with the corresponding Gaussian value as 0.01 . Therefore, when evaluating the accessibility of prehospital emergency medical services, value $\beta$ should be set at least to 10 so that the Gaussian value is always greater than 0.01 . Each slope of the distance decay weight change image is close to 0.5 when location $\beta$ is reassigned to 85 . In addition, when value $\beta$ continues to increase, the change in the distance decay function will be greatly gentle, and the decay effect of time distance on the first aid accessibility is relatively weak, so 85 can be determined as the maximum value of the coefficient. When the SD3SFCA model is used to measure the accessibility of prehospital emergency services, value $\beta$ should be within the range of 10 to 85 and the specific value should be further determined according to the actual situation of the study area.

\subsection{Quantitative Analysis of Supply-Demand Difference.} In the model, the supply capacity $S$ of emergency stations and the demand degree $P$ of demand points are quantitatively determined by selecting representative indexes and assigning different index weights. The specific index selection is shown in Table 1.

Similar to other public service facilities, the supply capacity of emergency stations is directly related to their scale. Infrastructure supply capacity and technical quality supply capacity jointly determine the scale of the emergency station. Therefore, the index of emergency station supply capacity is mainly selected from these two aspects. Referring to the existing research results and combining them with the characteristics of prehospital first aid work, we have selected the number of ambulances $S_{f 1}$ and beds $S_{f 2}$ to indicate the infrastructure supply capacity of the first aid station $S_{f}$, and the level of hospital $S_{q 1}$ and the number of clinical specialties $S_{q 2}$ to indicate the technical quality supply capacity of the first aid station $S_{q}$. A large number of studies show that densely populated areas are more prone to emergencies and emergency needs and that once there is an emergency, it is easy to cause more adverse impacts on personal and social security. Therefore, in addition to permanent residents, we should focus on the first aid needs of schools, shopping malls, and subway stations. The quantitative indicators of the difference in demand degree are shown in Table 1, in which the resident population $P_{1}$, school attendance $P_{2}$, the average daily passenger flow of shopping malls $P_{3}$, and the average daily passenger flow at traffic stations $P_{4}$ are selected as the demand indicators for emergency medical services.

The supply capacity $S$ of each emergency station and the demand degree $P$ of residents' demand points $i$ are expressed as follows:

$$
\begin{aligned}
& S=S_{f}+S_{q}=w_{f 1} S_{f 1}+w_{f 2} S_{f 2}+w_{q 1} S_{q 1}+w_{q 2} S_{q 2} \\
& P=w_{i 1} P_{i 1}+w_{i 2} P_{i 2}+w_{i 3} P_{i 3}+w_{i 4} P_{i 4} .
\end{aligned}
$$

The entropy weight model, a typical objective weighting method, is used to determine the weights $w$ of supply and demand indicators in equations (5) and (6). Compared with the subjective weighting method, it excludes the influence of human factors and obtains a relatively stable weight, which has been widely used in the evaluation of the capacity of public service facilities. At the same time, the accessibility of prehospital emergency care is directly affected by supply and demand indicators and what is needed is a data-oriented weighting method that reflects the objective status of supply 
TABLE 1: Indicators of supply and demand of prehospital emergency medical services.

\begin{tabular}{|c|c|c|}
\hline Target layer & First-level indicator layer & Second-level indicator layer \\
\hline $\begin{array}{l}\text { The supply capacity of prehospital emergency } \\
\text { medical services } S\end{array}$ & $\begin{array}{l}\text { The supply capacity of infrastructures } \\
S_{f} \\
\text { The supply capacity of technical quality } \\
S_{q}\end{array}$ & $\begin{array}{l}\text { The number of ambulances } S_{f 1} \\
\text { The number of hospital beds } S_{f 2} \\
\text { Hospital level relied on } S_{q 1} \\
\text { The number of clinical specialties } S_{q 2}\end{array}$ \\
\hline $\begin{array}{l}\text { The demand of prehospital emergency medical } \\
\text { services } P\end{array}$ & $\begin{array}{l}\text { Demand level of other densely } \\
\text { populated areas }\end{array}$ & $\begin{array}{c}\text { Resident population } P_{1} \\
\text { School attendance } P_{2} \\
\text { Average daily passenger flow of shopping } \\
\text { malls } P_{3} \\
\text { Average daily passenger flow at traffic } \\
\text { stations } P_{4}\end{array}$ \\
\hline
\end{tabular}

and demand levels. The low positive correlation between supply and demand indicators can be better applied to the entropy weight model, which makes the quantification of supply and demand differences more objective oriented and makes it possible to directly compare the accessibility of different regions.

Essentially, the first step of the SD3SFCA model reflects the service availability of each emergency site for each emergency demand point from an omniscient perspective, the second step obtains the potential service capacity of each emergency site affected by dispatching alternatives, and the third step summarizes the ability of each demand point to obtain prehospital emergency service. In fact, it is a realistic assumption that the supply-demand points are regarded as a competitive relationship during estimation of the accessibility level of prehospital emergency services because when adjacent emergency stations are also available, people's demand for an emergency station will decrease accordingly. However, the addition of this competitive relationship may not fully conform to the gravity model, so setting up an evaluation program is a necessity for testing the effect of the SD3SFCA model in measuring accessibility. Meanwhile, the SD3SFCA model is an improvement built on the original floating catchment area model. Hence, it is necessary to compare it with other widely used floating catchment area models to judge the progress of the improved one in practical application. In the next section, a case will be adopted to evaluate the performance of the SD3SFCA model to determine whether it is suitable for the measurement of the accessibility level of prehospital emergency medical services.

\section{Study Areas and Data Resources}

Taking 12 subdistricts in the West Coast New Area of Qingdao as examples, this study tends to analyze and evaluate the effectiveness and progress of the SD3SFCA model. With the application of ArcGIS10.6 software, the administrative division map of the West Coast New Area fulfills the vectorization. Besides, the study area is divided into 303 traffic districts with the centroid of each traffic district taken as the coordinate of demand point. In this study, the division method of the traffic district is coordinated with the general urban traffic planning; i.e., based on the principle of homogeneity and uniformity, the traffic district is divided according to the physical barrier of trunk roads, ecological barrier of rivers and mountains, large residential communities, and other group boundaries. Four types of densely populated areas, such as residential areas of the street community in the West Coast New Area, public schools, shopping districts, and traffic sites, are selected as the study units to establish the basic geographic database including population information (see Figure 2).

The demographic data used in the case study are analyzed by using Baidu LBS location data. Each geographical location is replaced by its geometric center, and the medical data concerned with emergency facilities are derived from the official websites of the Qingdao emergency center, the West Coast New Area emergency center, and the health information network. Road network traffic data are collected from open source data provided by OpenStreetMap. The traffic network data set (see Figure 3 ) is established, and the relevant data of the selected densely populated points and the medical data of the emergency center are imported into ArcGIS.

\section{Evaluation of the SD3SFCA Model}

The performance evaluation of the SD3SFCA model mainly includes two parts: effectiveness evaluation and progress evaluation. In the effectiveness evaluation, the SD3SFCA model is used to calculate the spatial accessibility of prehospital emergency medical services in the West Coast New Area with visualized results. The stability of the results is obtained by testing the accessibility changes of the impedance coefficient under different distances; the accessibility results of different traffic situations are used to test whether the improved model can reasonably distinguish the accessibility level of prehospital emergency medical services in different regions, so as to verify its effectiveness. In progress evaluation, the accessibility of prehospital emergency medical services of the new model is compared with other evaluation models to verify its progress.

\subsection{Effectiveness Evaluation of the SD3SFCA Model}

5.1.1. Accessibility Assessment under Different Impedance Coefficients. In this study, different impedance coefficient values are selected to calculate the spatial accessibility of prehospital emergency medical services in the case area. The stability of calculation results of the SD3SFCA model is 


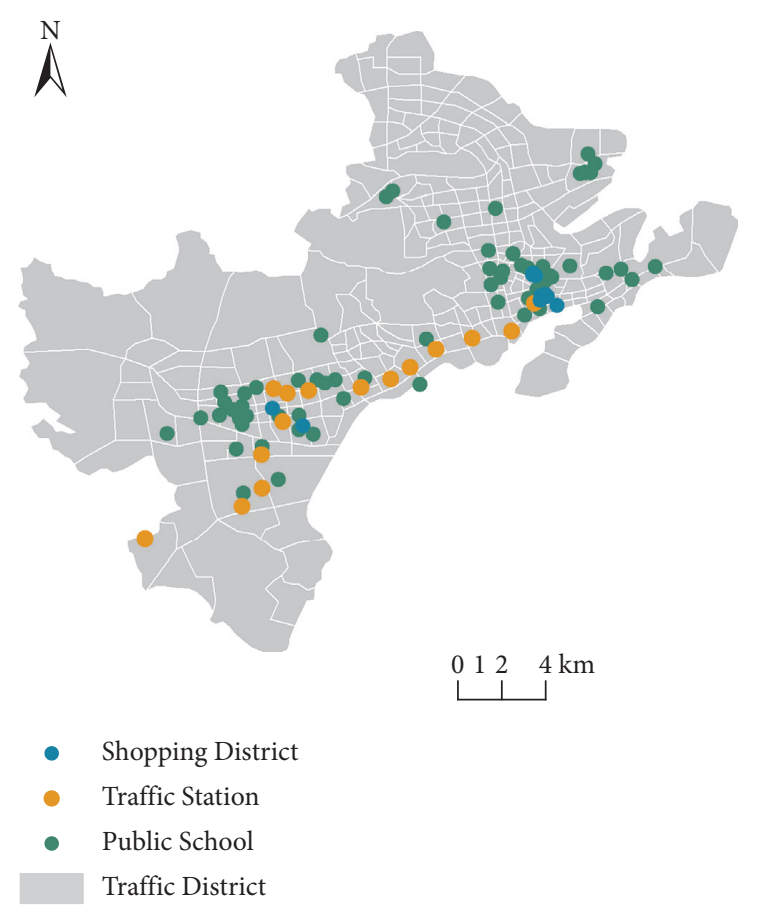

FIGURE 2: A distribution map of densely populated points and traffic districts.

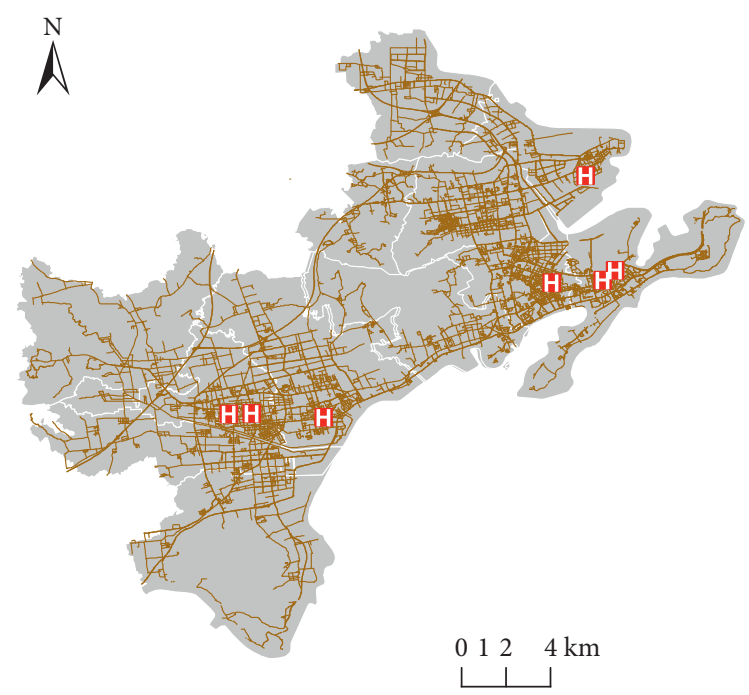

[H Emergency Station

— Road Network

- Administrative Division

Figure 3: A distribution map of traffic networks and emergency stations.

evaluated by the changes in geographic patterns of accessibility results. Figure 4 shows the calculation results of SPAI with six distance impedance coefficients $\beta$ (10-85, in increments of 15). The results are divided into six levels with the same interval for visual presentation so that the accessibility under different coefficients can be directly compared. The higher the SPAI, the higher the accessibility levels in this area, which means the accessibility of prehospital emergency medical services is higher. As is shown in Figure 4, the geographic spatial patterns of SPAI have changed significantly with the first two impedance coefficients $(\beta=10$, $\beta=25)$. With the increase in the coefficients, the accessibility levels generally increase, except in the areas that cannot be reached by emergency stations within 15 minutes. After the third distance impedance coefficients, the accessibility levels of each region in the geographic spatial patterns remain basically unchanged and tend to be stable.

Figures 5 and 6 further illustrate the trend of SPAI stability. The statistics of standard deviations and means with different impedance coefficients $\beta$ are shown in Figure 5. The means is not very different in general, while the standard deviations show a significant downward trend. The decline of standard deviations means that the dispersion of SPAI values is reduced and the value distribution is more concentrated. The standard deviations drop sharply in the first two impedance coefficients $(\beta=10, \beta=25)$, and then the downward trend slows down. Figure 6 shows the changes in the distribution of the accessibility levels in each area with different impedance coefficients $\beta$. Figure 6 visually presents that the attenuation effect of time distance on prehospital emergency services is weakened and the spatial distribution of accessibility is more balanced. When $\beta=10$, the strong distance decay leads to a large difference in accessibility levels and the highest level I and the lowest level VI of accessibility have regional distribution. With the increase in $\beta$, the distribution of the accessibility levels becomes more balanced and the areas at the two ends are concentrated on the middle levels III and IV. The variation trend of the standard deviations in Figure 5 corresponds to the change in the accessibility levels in Figure 6. Smaller $\beta$ causes a greater difference in accessibility between communities. While $\beta$ continues to increase, the difference gradually shrinks and stabilizes, related to the decrease in the effect of distance decay with the increase in $\beta$.

The changes in the distribution of SPAR and ASPA with different impedance coefficients are shown in Figures 7 and 8. It can be observed that the changes in the geographic patterns of SPAR and ASPA are similar to those of SPAI. The geographic patterns change significantly with the first two impedance coefficients. The accessibility levels of each area increase significantly except for the areas that cannot be reached by the emergency station within 15 minutes. However, from the third distance impedance coefficient, the accessibility levels of each region do not change. This means that the SPAI value calculated by the SD3SFCA model can show the stability characteristics of SPAR and ASPA.

In order to further determine whether the SPAI results obtained by the improved model can reflect supply-demand characteristics of the study area, we select 16 coefficient values (10-85, in increments of 5) from the range of distance impedance coefficients determined in the previous section and perform one-way analysis of variance between SPAI and ASPA results under 16 coefficient values. We assume $\mathrm{H} 0$ : $A_{\mathrm{SPAI}, \beta_{1}} / A_{\mathrm{ASPA}, \beta_{1}}=A_{\mathrm{SPAI}, \beta_{2}} / A_{\mathrm{ASPA}, \beta_{2}}=\ldots=A_{\mathrm{SPAI}, \beta_{i}} / A \mathrm{ASPA}$, $\beta_{i}=1$, where $\beta_{i}$ represents the value of the $\mathrm{i}$-th distance impedance coefficient. Similar to the expected result, under the significance level limit of 0.05 , the test accepts $\mathrm{H} 0$ 


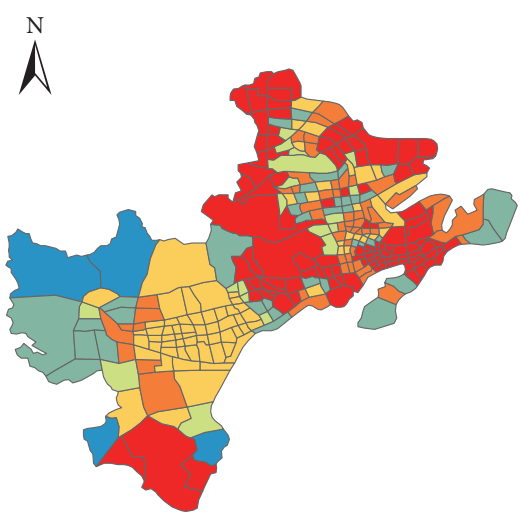

SPAI

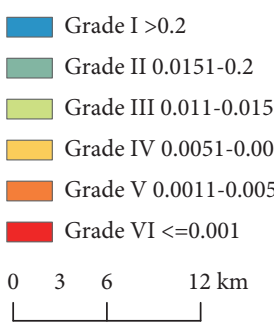

(a)

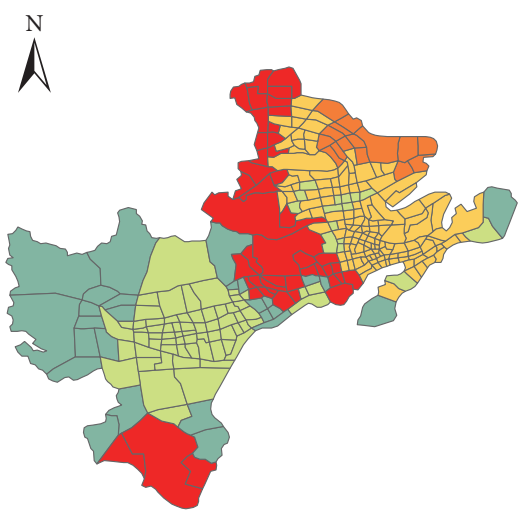

SPAI

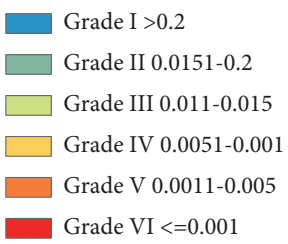

$\begin{array}{llll}0 & 3 & 6 & 12 \mathrm{~km}\end{array}$

(d)
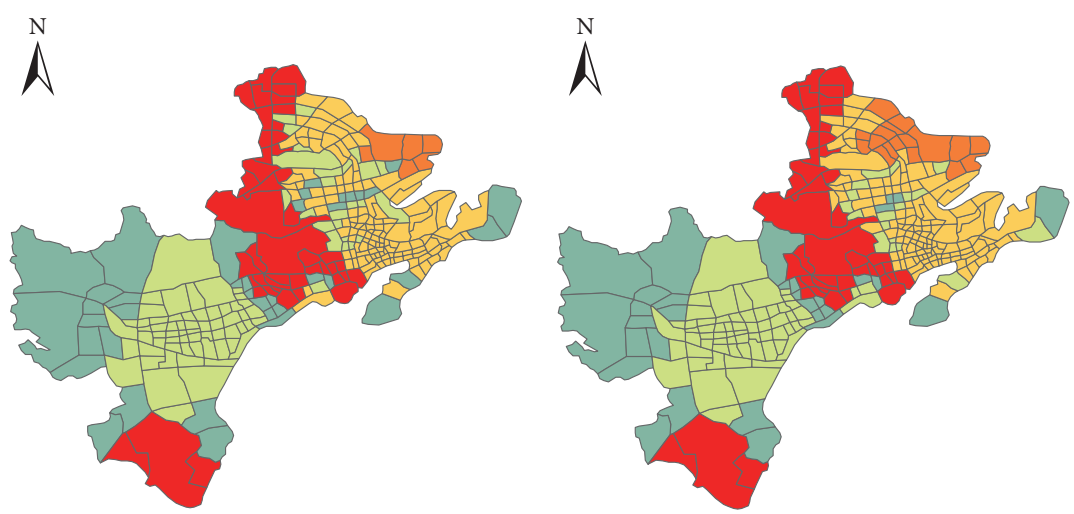

SPAI

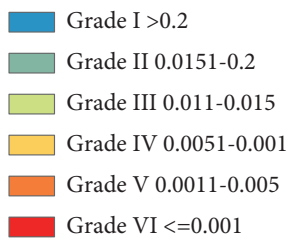

SPAI

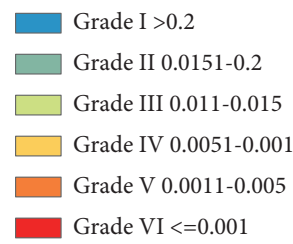

$\begin{array}{llll}0 & 3 & 6 & 12 \mathrm{~km}\end{array}$

(c)

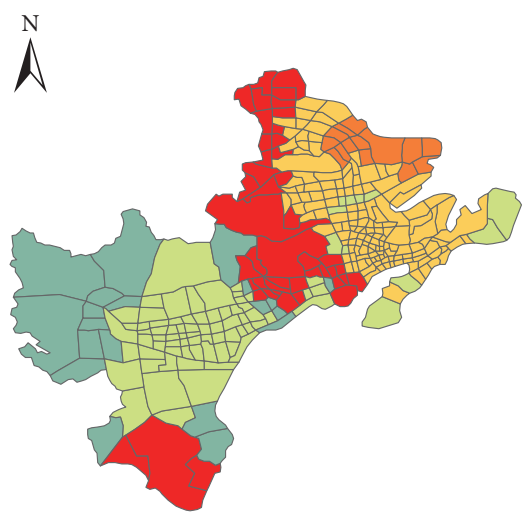

SPAI
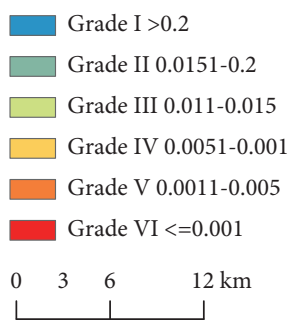

(e)

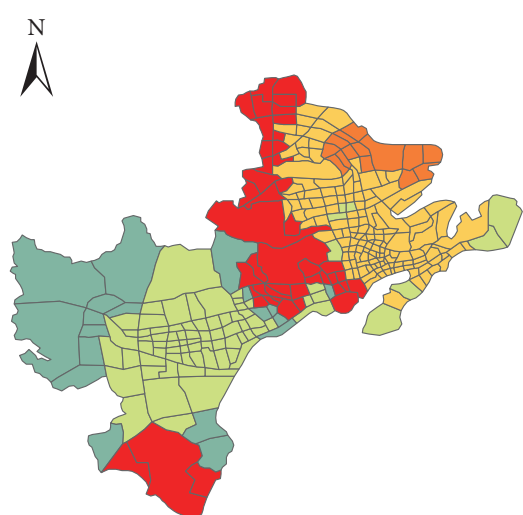

SPAI

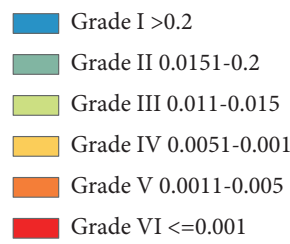

$\begin{array}{llll}0 & 3 & 6 & 12 \mathrm{~km}\end{array}$

(f)

Figure 4: The geographic patterns of spatial access index (SPAI) with different impedance coefficients. (a) $\beta=10$. (b) $\beta=25$. (c) $\beta=40$. (d) $\beta=55$. (e) $\beta=70$. (f) $\beta=85$.

$(F=0.098, p=0.758)$. There is a significant similarity between SPAI and ASPA values, which is more intuitively shown in Figure 9. SPAI calculated by the improved model can not only show good stability but also achieve the effect of ASPA reflecting the actual supply-demand ratio and identifying the shortage area. It has a significant advantage in the measurement of the accessibility of prehospital emergency medical services.
5.1.2. Accessibility Assessment with Different Traffic Scenarios. Theoretically, emergency vehicles are not restricted by signal lights and road speed limits in response operations, but in actual traffic, they will be affected by the safety of traffic and other vehicles. Therefore, the actual traffic situation cannot be ignored in the assessment of the level of emergency medical services. We analyze the accessibility levels of emergency medical services under three 


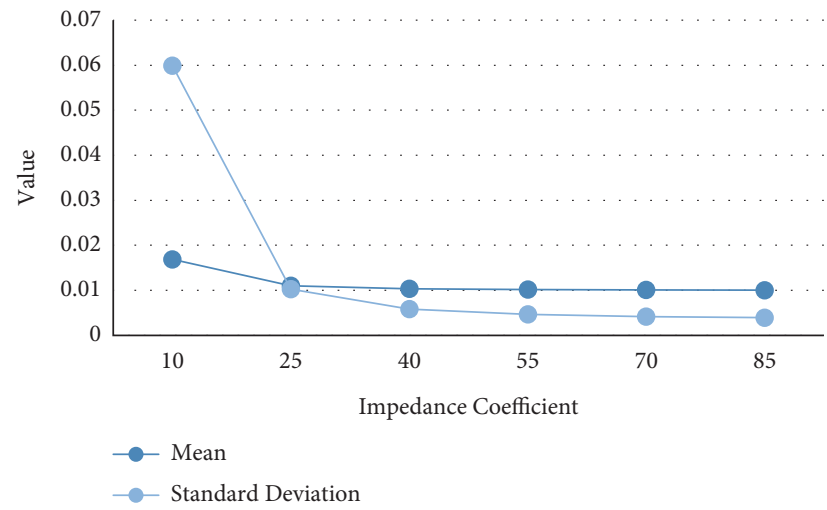

FIgURE 5: The standard deviations and means of SPAI with different impedance coefficients.

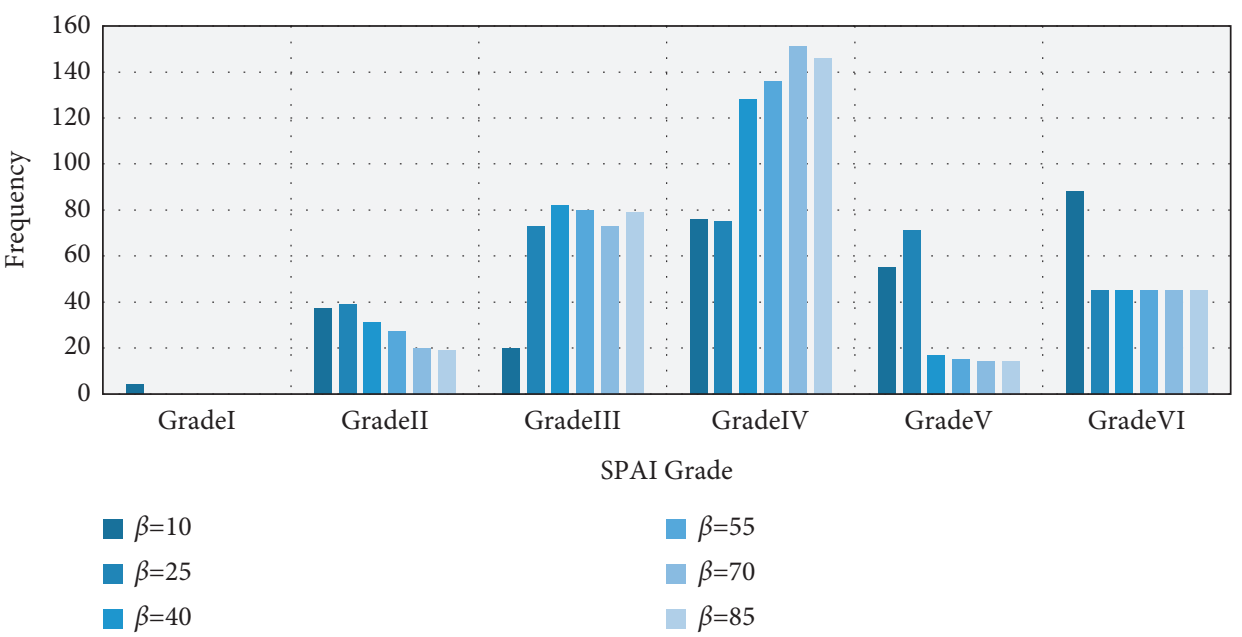

FIgURE 6: The distribution of SPAI grades with different impedance coefficients.

traffic scenarios of a peak, flat peak, and free flow and simulate different traffic scenarios by setting different speeds of emergency vehicles between emergency stations and demand points. The average speed of other vehicles in peak and flat peak scenarios provided by the Baidu map developer platform is taken as the speed of emergency vehicles, and the speed of emergency vehicles in the free flow scenario is set at $80 \mathrm{~km} / \mathrm{h}$ in consideration of driving safety in response operations. The accessibility calculated by different road speeds is not directly comparable. Thus, in this paper, according to the level of accessibility of demand points, the demand points are divided into 6 levels based on the method of natural breakpoints and the changes of accessibility are analyzed and compared through the changes of levels. When $\beta=25$, it represents a relatively strong time distance impedance effect. Therefore, in the comparative analysis of the accessibility of prehospital emergency medical services in different traffic scenarios, considering the characteristics of high effectiveness of emergency medical services, we set $\beta$ to 25.

The geographic patterns of accessibility of prehospital emergency medical services in different periods are shown in Figure 10. The main changes in the accessibility levels during peak and flat peak periods are reflected in the area around the emergency stations of Changjiang Road Subdistrict and Xuejiadao Subdistrict in the east of the study area, due to the large difference in road traffic speed between flat peak and peak periods in this area. It can be concluded that traffic congestion increases the accessibility of communities near emergency stations and that the difference in accessibility of emergency medical services between traffic districts has also expanded accordingly. This is because, due to the reduction in traffic speed, the competition between the districts around the emergency stations and the longer-distance traffic districts is correspondingly reduced.

In free flow condition, the traffic speed is set to $80 \mathrm{~km} /$ $h$ in consideration of traffic safety in emergency vehicle response operations. As is seen from the visualization results, the southernmost Dazhu Mountain Scenic Area still cannot receive emergency medical services within 15 minutes, while other areas maintain a high level of accessibility. It is worth noting that the accessibility levels around the emergency station in the southwest of the study area have decreased compared with the flat peak period. This is because the model fully considers the differences in the supply-demand of prehospital emergency medical services. The increase in road traffic speeds increases the potential first aid demand undertaken by 


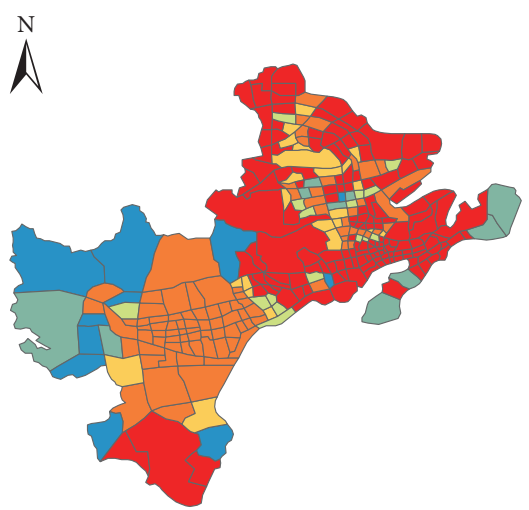

SPAR
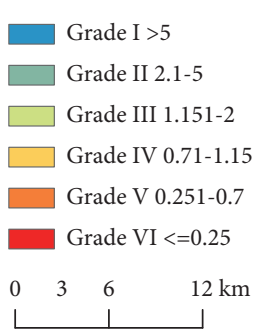

(a)

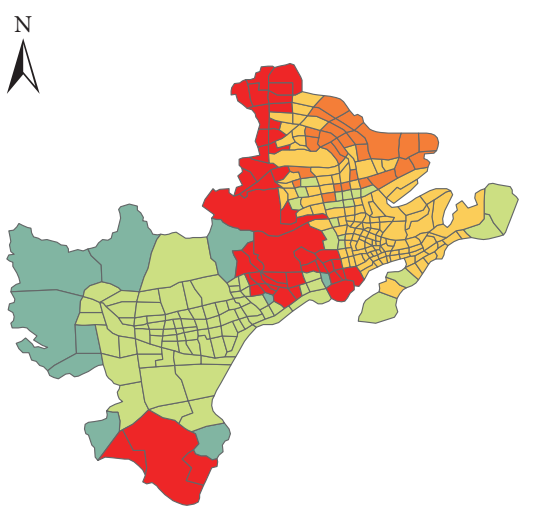

SPAR

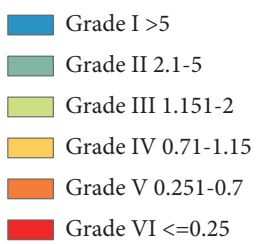

$\begin{array}{llll}0 & 3 & 6 & 12 \mathrm{~km}\end{array}$

(d)

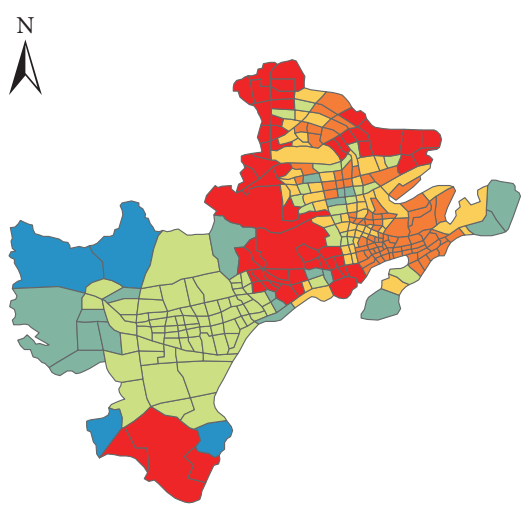

SPAR

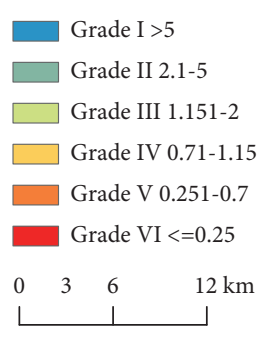

(b)

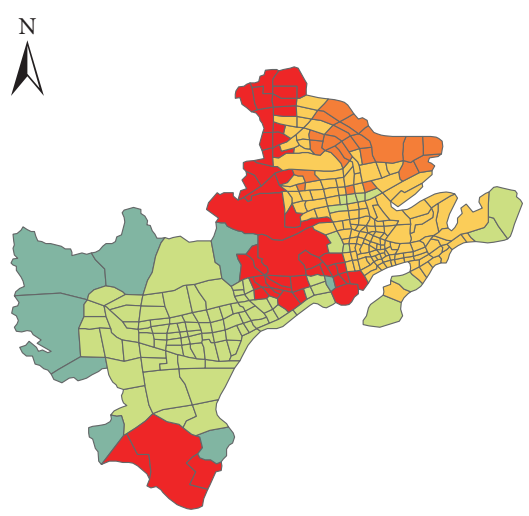

SPAR

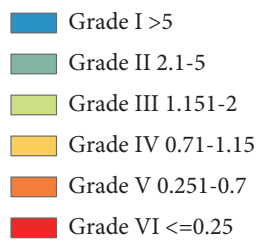

$\begin{array}{llll}0 & 3 & 6 & 12 \mathrm{~km}\end{array}$

(e)

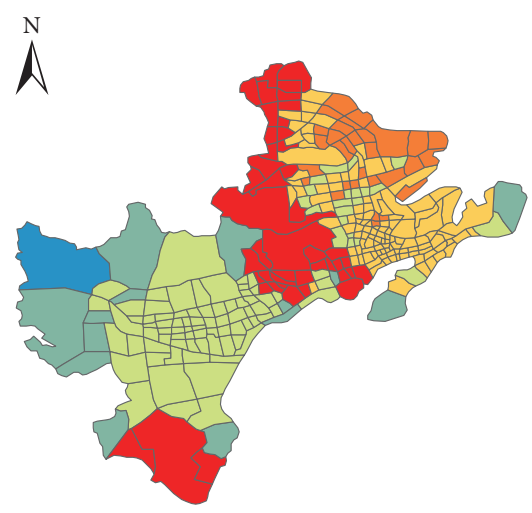

SPAR

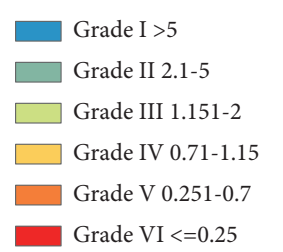

$\begin{array}{llll}0 & 3 & 6 & 12 \mathrm{~km}\end{array}$

(c)

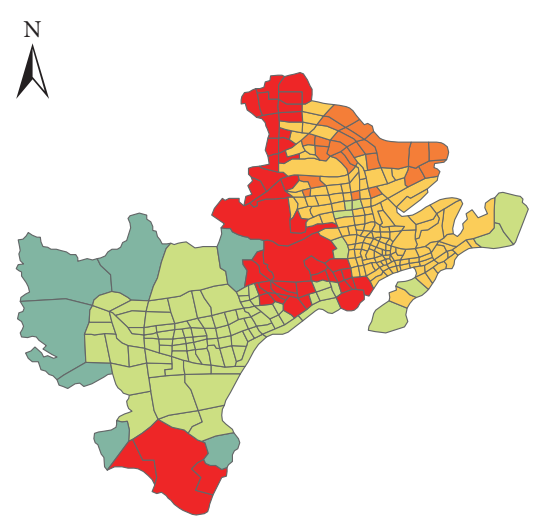

SPAR

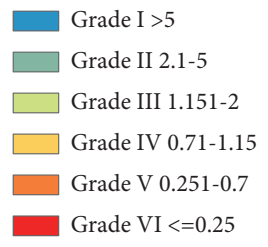

$\begin{array}{llll}0 & 3 & 6 & 12 \mathrm{~km}\end{array}$

(f)

FIGURE 7: The geographic patterns of spatial access ratio (SPAR) with different impedance coefficients. (a) $\beta=10$. (b) $\beta=25$. (c) $\beta=40$. (d) $\beta=55$. (e) $\beta=70$. (f) $\beta=85$.

emergency stations in the area, and the limited supply capacity reduces the remaining service capacity. Through the analysis of the spatial accessibility of prehospital emergency medical services under the three traffic scenarios of peak, flat peak, and free flow, it can be seen that the SD3SFCA model can distinguish the accessibility under different traffic scenarios and can reflect the impact of traffic congestion on accessibility.
5.2. Progress Evaluation of the SD3SFCA Model. In previous studies, models such as 2SFCA, E2SFCA, and 3SFCA have been widely used in the evaluation of public service levels such as medical care, and their effectiveness has been proved. However, they overestimate, underestimate, or even ignore the supply capacity and demand for public services. In order to overcome these shortcomings, this study has conducted further research on this basis and proposed the SD3SFCA 


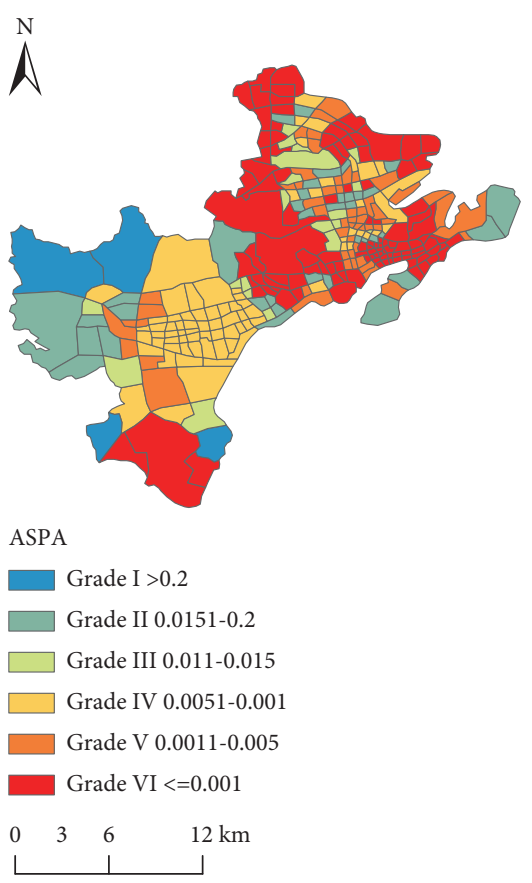

(a)

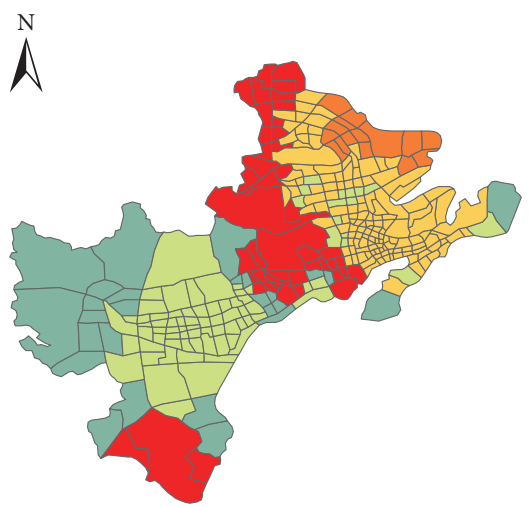

ASPA

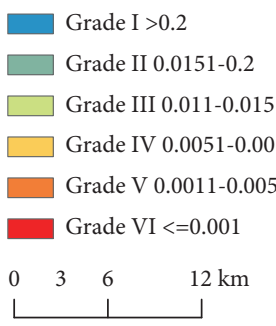

(d)
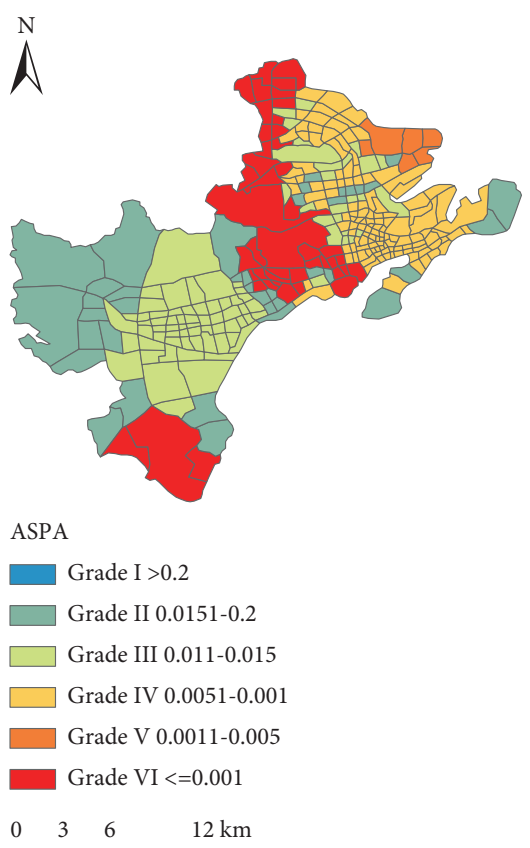

(b)

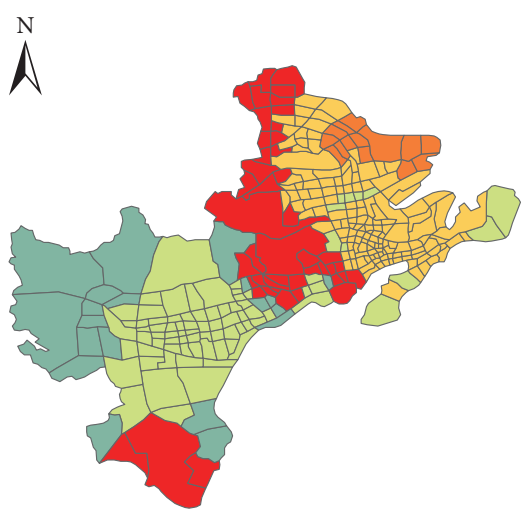

ASPA

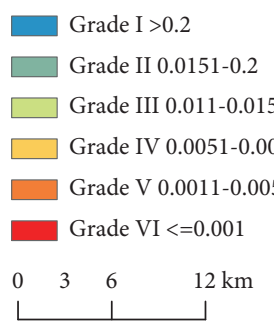

(e)

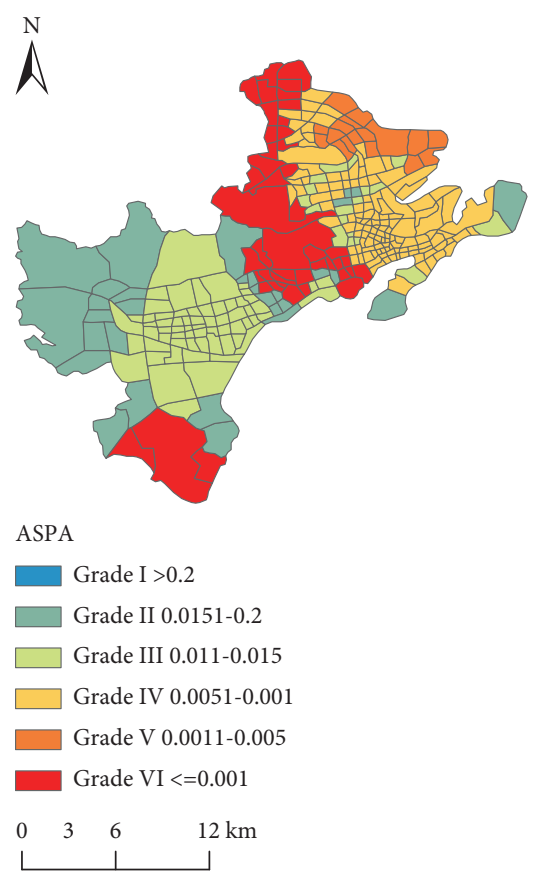

(c)

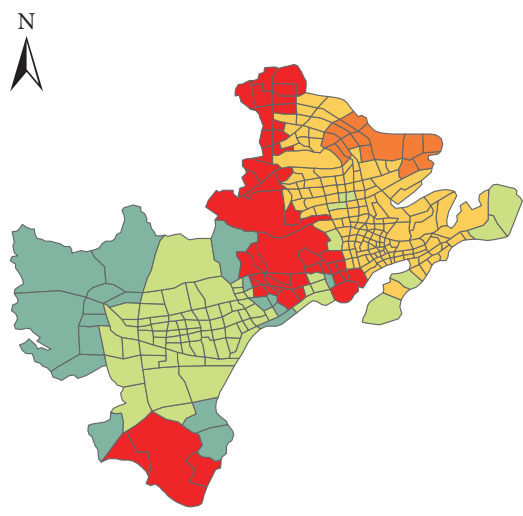

ASPA

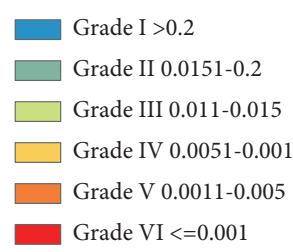

$\begin{array}{llll}0 & 3 & 6 & 12 \mathrm{~km}\end{array}$

(f)

FIGURE 8: The geographic patterns of adjusted spatial access (ASPA) with different impedance coefficients. (a) $\beta=10$. (b) $\beta=25$. (c) $\beta=40$. (d) $\beta=55$. (e) $\beta=70$. (f) $\beta=85$.

model considering the difference between supply and demand. Therefore, the 2SFCA, E2SFCA, 3SFCA, and SD3SFCA models are selected to calculate the spatial accessibility levels of the case area, and a comparative analysis is made from the three aspects of supply capacity, demand level, and SPAI to prove the progress of the SD3SFCA model in evaluating the accessibility of prehospital emergency medical services.
Figure 11 shows different quantification methods of supply capacity of first aid facilities. Compared with the method expressed on the same scale, the SD3SFCA model contains a supply index which can represent corresponding characteristics of emergency medical services and clearly distinguishes the supply capacity of each emergency facility. For example, relying on the No.3 first aid station of Xuejiadao Hospital, the quantitative value of its supply capacity 


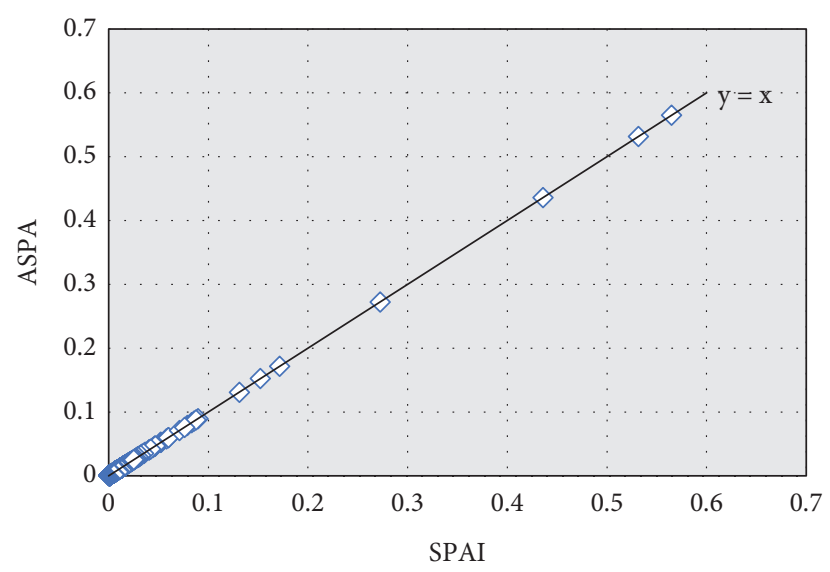

FIgUre 9: The value distribution of SPAI and ASPA.

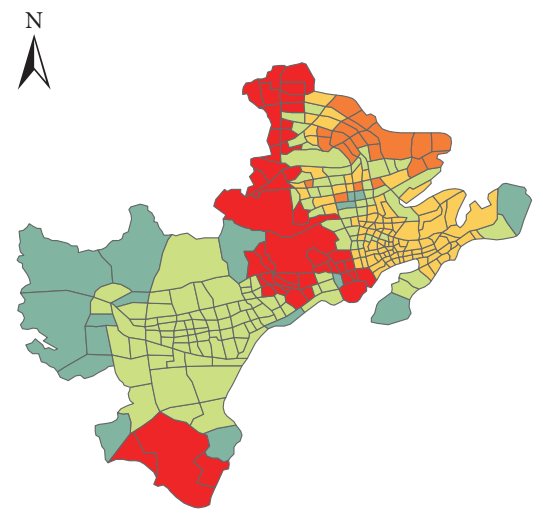

SPAI

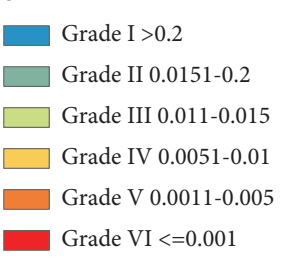

$0248 \mathrm{~km}$

$\mathrm{L} \perp$

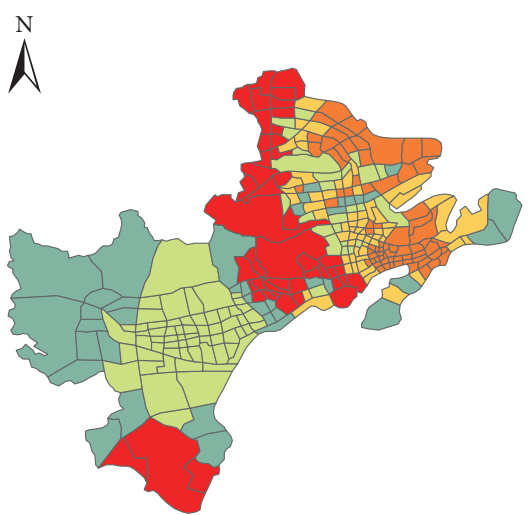

SPAI

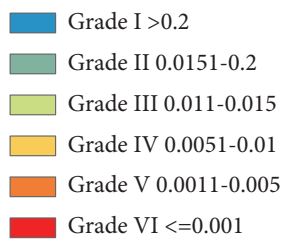

$0248 \mathrm{~km}$

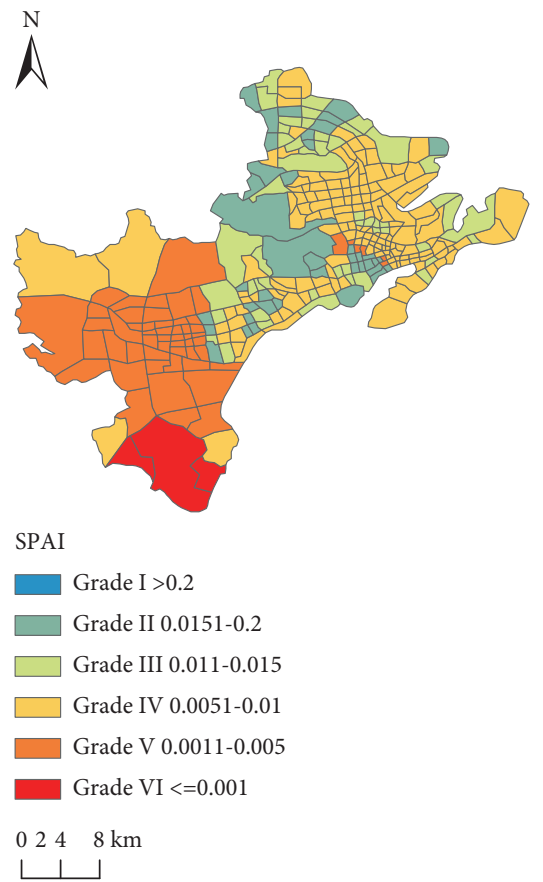

(c)

(a)

(b)

Figure 10: The geographic patterns of accessibility of prehospital emergency medical services with different traffic scenarios. (a) Peak. (b) Flat peak. (c) Free flow.

is obviously lower than that of other emergency facilities, due to the relatively weak hardware and low medical level in Xuejiadao Hospital. Compared with the quantitative method of expressing the supply capacity by the number of beds, the numerical difference of the supply capacity of each first aid facility in the SD3SFCA model is reduced, because the addition of different indexes avoids overestimation of the supply capacity difference.

As is shown in Figure 12, the SD3SFCA model takes into account the emergency needs of typical densely populated areas, which is significantly different from the previous method of using residential population to indicate the degree of demand. In the method of quantifying the resident population, the degree of demand varies greatly from region to region and most regions have higher levels of demand, showing a strong demand for emergency medical care. In the SD3SFCA model, although the total amount of population data increases, the difference of overall demand degree decreases, which indicates that the empowerment of the data of shopping mall passenger flow, school attendance, and subway passenger flow enriches its consideration conditions while alleviating the overestimation of demand.

As is shown in Figure 13, the accessibility of prehospital emergency medical services in the study area derived from the four models of 2SFCA, E2SFCA, 3SFCA, and SD3SFCA is visualized. The geographic distribution pattern calculated 


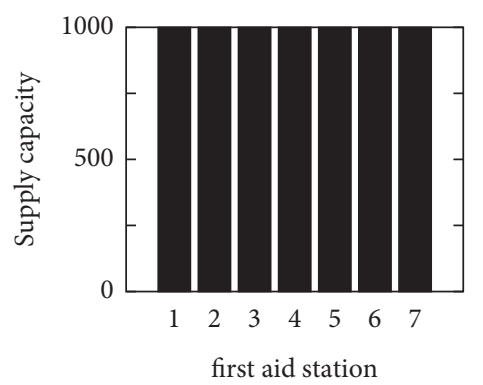

(a)

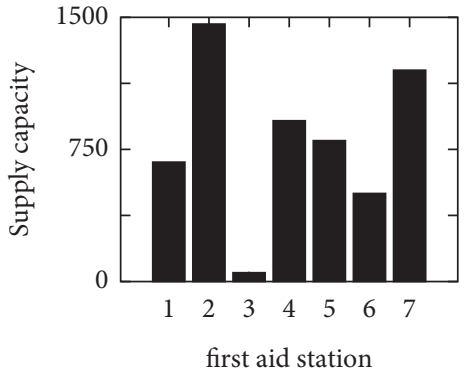

(b)

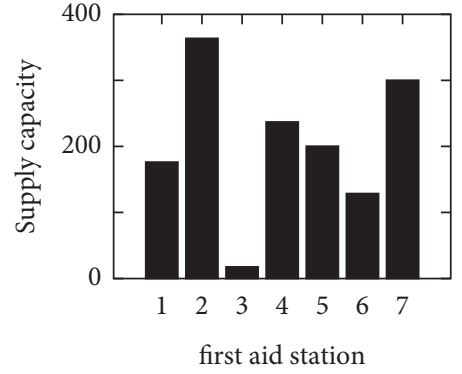

(c)

Figure 11: The different representations of first aid stations supply capacity. (a) Fixed numerical representation. (b) Number of beds representation. (c) SD3SFCA model representation.
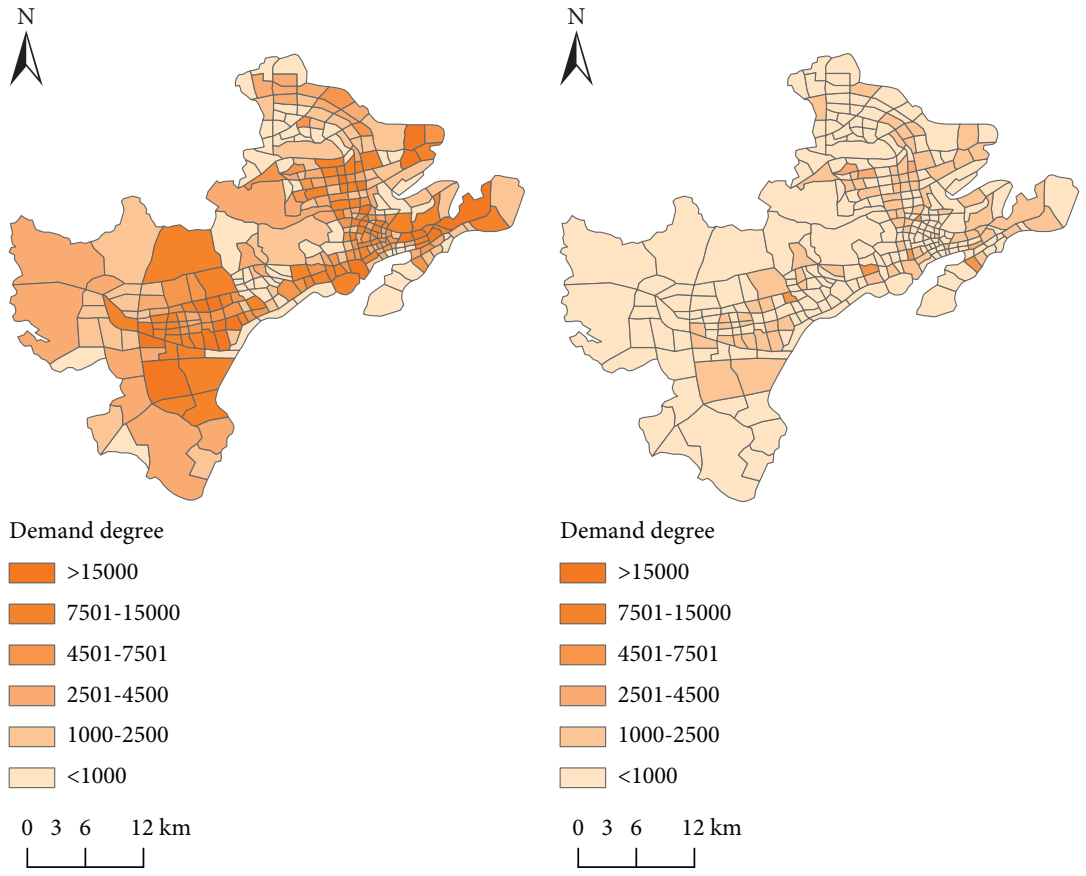

(a)

(b)

FIgURE 12: The different representations of degree for prehospital emergency care. (a) Demand degree is indicated by the number of living populations. (b) Demand degree is expressed in the SD3SFCA model.

by the 2SFCA model is extremely regular, and the corresponding SPAI gradually decreases with the emergency station as the center. The reason for the high level of accessibility at the junction of Changjiang Road Subdistrict and Xin'an Subdistrict is that the 2SFCA model ignores the influence of distance impedance. It does not meet the requirement of time sensitivity of emergency medical services and overestimates the remaining service capacity of lowlevel emergency stations. Because these two methods take into account the influence of distance impedance, the spatial distribution patterns of accessibility by the E2SFCA model and the 3SFCA model show great similarity, but both methods have low levels of emergency medical service space around the emergency station. However, as the distance from the emergency station increases, SPAI increases, because these two methods overestimate the level of demand for emergency medical services.

The spatial distribution pattern of accessibility of the SD3SFCA model proposed in this study is different from the above three results. Compared with the 2SFCA model, the accessibility levels around the hospital are relatively low because the SD3SFCA model fully takes into account the impact of the distance attenuation of emergency medical services and differently quantifies the supply capacity of emergency stations. Compared with the E2SFCA and 3SFCA models, the difference in accessibility level is small on the whole. Except for the range that emergency medical services cannot reach within 15 minutes, the accessibility of lowgrade areas decreases because the SD3SFCA model simulates the scheduling process of the emergency center through 


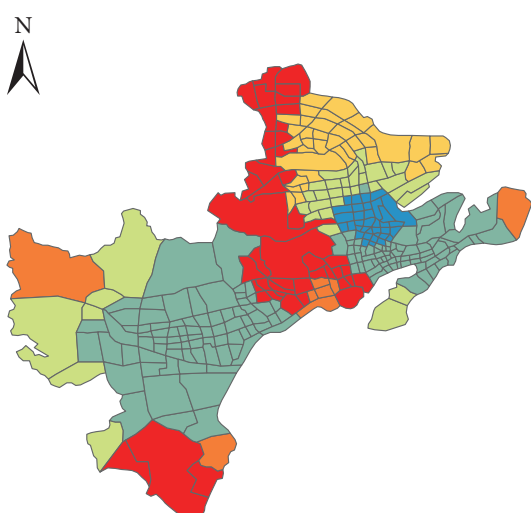

SPAI

Grade I

$\square$ Grade II

$\square$ Grade III

$\square$ Grade IV

$\square$ Grade V

Grade VI

$\begin{array}{llll}0 & 3 & 6 & 12 \mathrm{~km}\end{array}$

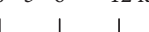

(a)
$\stackrel{N}{N}$

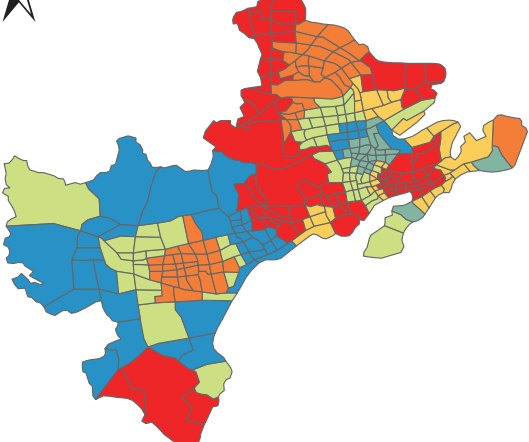

SPAI

$\square$ Grade I

$\square$ Grade II

$\square$ Grade III

$\square$ Grade IV

$\square$ Grade V

Grade VI

$\begin{array}{llll}0 & 3 & 6 & 12 \mathrm{~km}\end{array}$

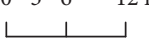

(b)

$\stackrel{N}{N}$
$\stackrel{N}{N}$

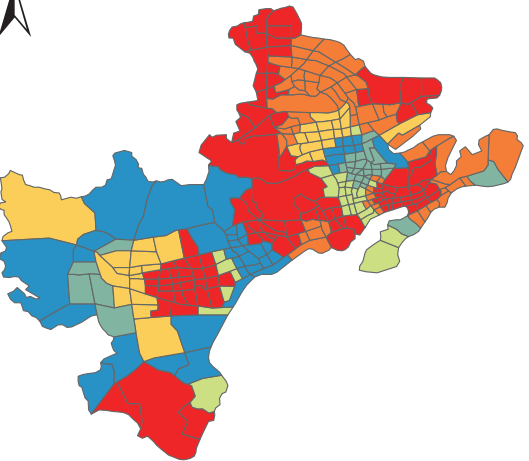

SPAI

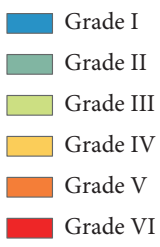

$\begin{array}{llll}0 & 3 & 6 & 12 \mathrm{~km}\end{array}$

(c)

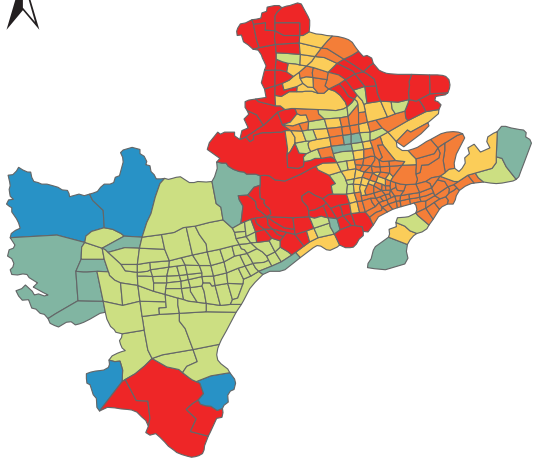

SPAI

$\square$ Grade I

$\square$ Grade II

$\square$ Grade III

$\square$ Grade IV

$\square$ Grade V

Grade VI

$\begin{array}{llll}0 & 3 & 6 & 12 \mathrm{~km}\end{array}$

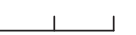

(d)

FIGURE 13: The different representations of degree for prehospital emergency care. (a) 2SFCA, (b) E2SFCA, (c) 3SFCA, and (d) SD3SFCA. 
selection probability to avoid overestimation of demand for first aid. The comparative analysis of different models can prove that the SD3SFCA model is closer to the actual workflow of prehospital emergency medical services and can clearly differentiate the supply capacity and demand level of prehospital emergency medical services.

\section{Conclusion and Future Work}

This paper proposes an SD3SFCA model to measure the accessibility level of prehospital emergency medical services more reasonably from the perspective of supply and demand and takes the West Coast New Area of Qingdao as an example to prove its effectiveness and progressiveness through comparative analysis. Specifically, this method differentially quantifies the supply and demand of prehospital emergency medical services and adjusts them through the weight index. Based on supply and demand, the selection probability is used to calculate the effective simulation of the emergency center scheduling process, which is closer to the prehospital emergency medical work process in real life while avoiding overestimation or underestimation of supply and demand.

In order to verify the validity of the model, this study incorporates the improved new model into calculating the spatial accessibility of the case area and visualizes the results. In addition, we have tested whether the improved model can reasonably distinguish the accessibility levels of emergency medical services in different areas through the accessibility analysis of different distance impedance coefficient settings and the accessibility analysis under different traffic scenarios. By changing the distance impedance coefficients to visually present the accessibility level, the results show that when the distance impedance was strong, the accessibility level increased from 5 grades to 6 grades and the change in interregional accessibility level was in line with the characteristics of time sensitivity of prehospital emergency medical services. As the distance impedance changes from 10 to 25 , the number of lowest accessibility classes drops from 88 to 45 . However, when the distance impedance is greater than 25, the number of lowest accessibility classes will remain unchanged as the value of distance impedance increases. This means that in the SD3SFCA model, the influence of the distance impedance reached equilibrium and the SPAI distribution of the model also showed greater stability, which could directly reflect the prehospital emergency medical service level of accessibility. In addition, traffic congestion restricts the rescue of traffic communities far away from emergency stations, thereby increasing the accessibility of districts near emergency stations. The SD3SFCA model is excellent in distinguishing the accessibility levels of different regions and is easy to understand. It can be used as an important tool to measure the accessibility of prehospital emergency medical services.

In comparison with the results of several models widely used in the past, it can be found that the SD3SFCA model has made significant progress. The main advantage of the SD3SFCA model lies in its effective simulation of the emergency center scheduling process and reasonable assumptions about supply and demand. Distinguishing the difference in the capacity of supply facilities while adjusting population demand is also an important reason for the stability of its spatial pattern of accessibility. Regarding the quantification of supply capacity, full consideration is given to the work characteristics of emergency services and availability of data access so that they have the potential for wide application. In terms of demand, the difference between the highest and lowest demands is reduced from 6 grades to 4 grades, effectively avoiding the problem of overestimation of demand. The demand for emergency medical services in densely populated areas has been clearly reflected, so the degree of demand can be better adjusted.

There are several issues worth special attention when using the SD3SFCA model in the actual assessment of the accessibility of prehospital emergency medical services, in spite of its significant advantages. First, the speed of emergency vehicles in response work can be more accurate. In this study, the average speed of road traffic in different periods is used to determine the search area for emergency medical services. However, in the actual driving process of the emergency vehicle, with no speed limit, the speed of the emergency vehicle and other vehicles are different, although it is affected by the friction between other vehicles. In the follow-up study, actual GPS data may be used to better set the speed value of the emergency vehicle. Second, the demand in different locations at different time periods also exhibits the feature of "tidal." For example, during the daytime of the working day, the population density of the school area is high, while the population density of the residential areas at night is high. In future applications, the tidal feature of emergency demand can also be taken into consideration. In addition, it is necessary to set the value of the distance impedance coefficient more reasonably in actual evaluation. The values of the coefficients in this article are basically set for the purpose of comparison which basically conform to the characteristics of first aid. In order to more accurately assess the accessibility of emergency medical services in a certain area, the values should be set based on the survey data of the actual response work of prehospital emergency medical services.

Overall, this method has improved the model of the accessibility assessment of emergency medical services and provided a new perspective for it. The evaluation results are quite consistent with the actual situation. Because the SD3SFCA model is closer to the workflow of prehospital emergency treatment in real life, it can help relevant government departments identify the missing areas of prehospital emergency medical services more reasonably; meanwhile, it can analyze the causes and regular pattern of prehospital emergency service's shortage by different traffic speeds, supply capacities, and demand degrees in the same area. This method can effectively provide a decision-making basis to rationally allocate emergency medical resources, improve the level of prehospital emergency medical services, and ensure full coverage of prehospital emergency medical services. Therefore, it shows great application potential in the allocation and optimization of prehospital emergency medical resources. 


\section{Data Availability}

The data used to support the findings of this study are available from the corresponding author upon request.

\section{Conflicts of Interest}

The authors declare that there are no conflicts of interest regarding the publication of this paper.

\section{Acknowledgments}

This research was funded by the National Natural Science Foundation of China (71801144). In addition, it was also supported by the China Postdoctoral Science Foundation (2019M652437) and Shandong Province Postdoctoral Innovation Project (201903030).

\section{References}

[1] R. Aringhieri, M. E. Bruni, S. Khodaparasti, and J. T. van Essen, "Emergency medical services and beyond: addressing new challenges through a wide literature review," Computers \& Operations Research, vol. 78, pp. 349-368, 2017.

[2] M. Gulliford, J. Figueroa-Munoz, M. Morgan et al., "What does "access to health care" mean?" Journal of Health Services Research and Policy, vol. 7, no. 3, pp. 186-188, 2002.

[3] W. Hu, J. Tan, M. Li, J. Wang, and F. Wang, "Impact of traffic on the spatiotemporal variations of spatial accessibility of emergency medical services in inner-city Shanghai," Environment and Planning B: Urban Analytics and City Science, vol. 47 , no. 5, pp. 841-854, 2018.

[4] W. G. Hansen, "How accessibility shapes land use," Journal of the American Institute of Planners, vol. 25, no. 2, pp. 73-76, 1959.

[5] M. Yu and X. Yang, "Research on airport landside traffic service capacity base $\mathrm{d}$ on accessibility-take Hongqiao airport as an example," Intelligent Computer and Applications, vol. 9, no. 5, pp. 158-160+164, 2019.

[6] S. Freiria, A. O. Tavares, and R. P. Julião, "The benefits of a link-based assessment of health services accessibility: unveiling gaps in Central Region of Portugal," Land Use Policy, vol. 87, 2019.

[7] K. Kelobonye, H. Zhou, G. McCarney, and J. Xia, "Measuring the accessibility and spatial equity of urban services under competition using the cumulative opportunities measure," Journal of Transport Geography, vol. 85, Article ID 102706, 2020.

[8] W. Yu, K. Zhang, J. Li, H. Sun, and Y. Qu, "Urban public transport network accessibility based travel data," Journal of Transportation Systems Engineering and Information Technology, vol. 20, no. 4, pp. 106-112, 2020.

[9] L. Chen, J. Qian, T. Zhang, and J. Wang, “Accessibility to shopping malls in nanjing, China: comparative analysis with multiple transportation modes," Chinese Geographical Science, vol. 30, no. 4, pp. 710-724, 2020.

[10] L. Li, H. Ren, S. Zhao, Z. Duan, Y. Zhang, and A. Zhang, "Two dimensional accessibility analysis of metro stations in Xi'an, China," Transportation Research Part A: Policy and Practice, vol. 106, pp. 414-426, 2017.

[11] F. Wang and W. Luo, "Assessing spatial and nonspatial factors for healthcare access: towards an integrated approach to defining health professional shortage areas," Health \& Place, vol. 11, no. 2, pp. 131-146, 2005.

[12] C. Davy, S. Harfield, A. McArthur, Z. Munn, and A. Brown, "Access to primary health care services for indigenous peoples: a framework synthesis," International Journal for Equity in Health, vol. 15, no. 1, p. 163, 2016.

[13] G. Higgs, R. Zahnow, J. Corcoran, M. Langford, and R. Fry, "Modelling spatial access to general practitioner surgeries: does public transport availability matter?" Journal of Transport \& Health, vol. 6, pp. 143-154, 2017.

[14] S. Hou and H. Jiang, "An analysis on accessibility of hospitals in Changchun based on urban public transportation," Geographical Research, vol. 33, no. 5, pp. 915-925, 2014.

[15] $\mathrm{M}$. Tseng and $\mathrm{H}$. Wu, "Integrating socioeconomic status and spatial factors to improve the accessibility of community care resources using maximum-equity optimization of supply capacity allocation," International Journal of Environmental Research and Public Health, vol. 18, no. 10, 2021.

[16] Q. Wang and S. Gao, "Selection of emergency medical service based on accessibility calculation," Journal of Transportation Systems Engineering and Information Technology, vol. 19, no. 1, 2019.

[17] Y. Zhang, W. Li, H. Deng, Y. Li, and T. Liu, "Evaluation of public transport-based accessibility to health facilities considering spatial heterogeneity," Journal of Advanced Transportation, vol. 2020, Article ID 7645153, 10 pages, 2020.

[18] K. Shin and T. Lee, "Improving the measurement of the Korean emergency medical system's spatial accessibility," Applied Geography, vol. 100, pp. 30-38, 2018.

[19] W. Luo and F. Wang, "Measures of spatial accessibility to health care in a GIS environment: synthesis and a case study in the chicago region," Environment and Planning B: Planning and Design, vol. 30, no. 6, pp. 865-884, 2003.

[20] Y. Kim, Y. J. Byon, and H. Yeo, "Enhancing healthcare accessibility measurements using GIS: a case study in Seoul, Korea," PLoS One, vol. 13, no. 2, Article ID e0193013, 2018.

[21] W. Luo and Y. Qi, "An enhanced two-step floating catchment area (E2SFCA) method for measuring spatial accessibility to primary care physicians," Health \& Place, vol. 15, no. 4, pp. 1100-1107, 2009.

[22] N. Wan, B. Zou, and T. Sternberg, "A three-step floating catchment area method for analyzing spatial access to health services," International Journal of Geographical Information Science, vol. 26, no. 6, pp. 1073-1089, 2012.

[23] N. Wan, F. B. Zhan, B. Zou, and E. Chow, "A relative spatial access assessment approach for analyzing potential spatial access to colorectal cancer services in Texas," Applied Geography, vol. 32, no. 2, pp. 291-299, 2011.

[24] M. P. Kwan, "Space-time and integral measures of individual accessibility: a comparative analysis using a point-based framework," Geographical Analysis, vol. 30, no. 3, pp. 191-216, 1998. 